\title{
The Origin of Consciousness in the Universe.
}

\author{
Dr. Narayan Kumar Bhadra \\ L.S.S.S. High School (H.S); Gobardanga, North 24 Parganas, West Bengal, India.
}

\begin{abstract}
We study a series of new energy sources of the Gaussian energy group $S U(6), S U(12), S U(24)$, ....etc. called intelligences which created consciousness and which are responsible for the creation of everything of this universe. The effective quantity and quality of the consciousness of the earth-like planet where living elements were found depends on environment and place of position within the galaxy or cluster or super cluster etc. \& mechanical structure of the planet. The said energy sources created consciousness within the living cell and also controlled the whole universe with the symmetry breaking of the energy group $S U(11)[\supset$ $S U(6) \times S U(5) \times U(1)] ; S U(23) ; S U(47) ; \ldots \ldots$ etc. I. In the living body, generally consciousness controlled by the self gravitational force of the energy group $S U(6)$ and plays with biological revolution. But consciousness level of any living body including human group and also inanimate objects is particularly controlled by SU(12), $S U(24)$, ....etc. We examine the quantum measurement, using the Wheeler DeWitt wave equation over the complex space-time $R+i R_{I}$ of $(4+D)$ dimensions, where $D$ is an extra dimensions. We know that the quantum super-positions will continually be reaching the Diósi-Penrose $(D P)$ threshold for Objective Reduction in nonbiological settings as well as in biological ones, and usually take place in the purely random environment of a quantum system under measurement through the symmetry breaking of the Generalized Gaussian Energy Group from infinity i.e. from Big-Rip singularity when $R_{I} \rightarrow \infty$.
\end{abstract}

\section{Introduction:}

Masao Ito wrote that it may be take another half century to bring psychology into the field of neuroscience. This statement reflects the difficulty of extending knowledge about the pivotal role of the cerebellum from the domain of motor control to the cognitive domain. The shift of information processing from divergence to convergence in cerebella micro-complexes (Ito) parallels the shift from coherence to de-coherence in quantum information processing (LIoyd). Consciousness defines our existence: It was proposed by Roger Penrose and Stuart Hameroff in the mid of 1990's that consciousness depends on biologically 'orchestrated' quantum computations in collections of microtubules within brain neurons, that these quantum computations correlate with and regulate neuronal activity, and that the continuous Schrödinger evolution of each quantum computation terminates in accordance with the specific Dio'si - Penrose(DP) scheme of 'Objective Reduction' of the quantum state (OR). This orchestrated OR activity (Orch OR) is taken to result in a moment of conscious awareness and / or choice. This particular (DP) from of OR is taken to be a quantum-gravity process related to the fundamentals of space-time geometry with complex scale factor $R+i R_{I}$ in the large scale of the universe, where $\mathrm{R}$ is the 4-dimensional Einstein's universe and $i R_{I}(=a)$, the internal space-time of the extra-dimension 'D', which may be compared with the Kaluza-Klein cosmology. Hence solving the WDW equation of the wave function instead of Shrödinger's wave equation, we get a remarkable solutions of the wave function of the variable $R_{I}$ (where $R_{I}^{2}=-R^{2}$ ) in the tachyonic and pseudo-tachyonic universe, which assume as the source of intelligence or consciousness in the universe starting from Big-Rip singularity i.e. from the infinite space-time. Again Orch OR suggests a connection between brain bio-molecular processes and fine scale structure of the universe. There were three general assumption regarding the origin and place of consciousness in the universe have been commonly expressed as 1). Consciousness is not an independent quality but arose as a natural evolutionary consequence of the biological adaptation of brain and nervous system. 2). Consciousness is a quality that has always been in the universe. 3). Precursors of consciousness have always been in the universe; biology evolved a mechanism to convert conscious precursors to actual consciousness.

The quantum cosmology explain an appropriate quantum mechanical description of the universe, which was introduced and developed by DeWitt. In quantum cosmology the universe, as a whole is treated quantum mechanically and is described by a single wave function, $\Psi\left(\mathrm{h}_{\mathrm{ij}}, \Phi\right)$ defined on a manifold (super spaces) of all possible three geometries and all mater field configurations. The wave function $\Psi\left(\mathrm{h}_{\mathrm{ij}}, \Phi\right)$ has no explicit time dependence due to the fact that there is no real time parameter external to the Einsteins universe. Therefore, there is no Shrödinger's wave equation but the operator version of the Hamiltonian constraint of the Dirac canonical quantization procedure, namely vanishing of the variation of the Einstein-Hilbert action $\mathrm{S}$ with respect to the arbitrary lapse function $\mathrm{N}$. Thus $\mathrm{H}=\frac{\delta \mathrm{S}}{\delta \mathrm{N}}=0$, which is written as $\mathrm{H} \Psi\left(\mathrm{h}_{\mathrm{ij}}, \Phi\right)=0$. This equation is known as the Wheeler-DeWitt (WDW) equation. The goal of quantum cosmology by solving the WDW equation over 
the complex space $\left(\mathrm{R}+\mathrm{iR} \mathrm{R}_{\mathrm{I}}\right)$ is to understand the origin and source of consciousness and hence the intelligence, the evolution and the biological revolution of the universe.

According to modern quantum field theory, the structure of a vacuum turns out to be interrelated with some spontaneous symmetry breaking effects through the condensation of quantum fields, hence it is from infinity with some spontaneous symmetry breaking of the pseudo-tachyonic and tachyoic fields, it was shown that the energy pressure and density exists at Big-Rip singularity [Bhadra NK; THE COMPLEX QUANTUM AND CLASSICAL PSEUDO-TACHYONIC UNIVERSE; (IOSR-JM) e-ISSN: 2278-5728,p-ISSN: 2319765X, Volume 8, Issue 3 (Sep. - Oct. 2013), PP 15-32]. This phenomenon gives rise to non vanishing vacuum energy density of the form $<\mathrm{T}_{\mu \nu}>=-<\rho>\mathrm{g}_{\mu \nu}$; as for scalar fields.

Again our knowledge of the unification of the fields can guide us to determine how the fields in the vacuum state may be further organized. Although the high temperatures necessary for unification are physically absent in the vacuum, quantum physics indicates their presence in a virtual way. Due to uncertainty principle, as the dimension of space decrease, the equivalent energy per particle (i.e. temperature) increases. Also an estimate of the increase in equivalent temperature (energy/particle) can be done from the Heisenberg uncertainty relation. For simplicity let us consider only the $\mathrm{x}$ co-ordinate. If a particle (or a quantum of energy) is confined inside a cubical box a linear size $\Delta \mathrm{x}$, then the minimum uncertainty in the particle's momentum $\Delta \mathrm{p}_{\mathrm{x}}$ is given by the uncertainty principle: $\Delta \mathrm{x} . \Delta \mathrm{p}_{\mathrm{x}}=\mathrm{h}$ (Planck constant). A mass-less relativistic particle with the magnitude of momentum $\Delta \mathrm{p}_{\mathrm{x}}$ will have a kinetic energy E given by, $\Delta \mathrm{p}_{\mathrm{x}}=\frac{\mathrm{E}}{\mathrm{C}}$

The electro-weak unification occurs at an energy (per particle) of $200 \mathrm{GeV}$. The corresponding linear size of the box should be

$$
\Delta \mathrm{x}=\frac{\mathrm{h}}{\Delta \mathrm{p}_{\mathrm{x}}}=\frac{\mathrm{hc}}{\mathrm{E}} \sim 10-16 \mathrm{Cm} \text { and then } \mathrm{T}=10^{15} \mathrm{~K} .
$$

Similarly, $\Delta \mathrm{x} \sim 10^{-30} \mathrm{~cm}$. for $\mathrm{E}=10^{16} \mathrm{GeV}$ and $\mathrm{T}=10^{29} \mathrm{~K}$ and the Planck length $\Delta \mathrm{x} \sim 10^{-33} \mathrm{~cm}$ for $\mathrm{E}$ $=10^{19} \mathrm{GeV}$ and $\mathrm{T}=10^{32} \mathrm{~K}$. In a similar manner, we can calculate, $\Delta \mathrm{x} \sim 10^{-36} \mathrm{~cm}$ for $\mathrm{E}=10^{22} \mathrm{GeV}$ and so on. Note that as $\Delta \mathrm{x} \rightarrow 0$ then $\mathrm{E} \rightarrow \infty$ and also $\mathrm{T} \rightarrow \infty$.

\section{The Nature and Fundamental Problems of Quantum Mechanics:}

The term 'quantum' refers to a discrete element of energy in a system, such as the energy E of a particle, or of some other sub-system, this energy being related to a fundamental frequency $v$ of its oscillation, according to Max Planck famous formula (where $h$ is the Planck constant): $E=h v$. This deep relation between discrete energy levels and frequencies of oscillation underlies the wave/particle duality inherent in quantum phenomena. Neither the word 'particle' nor the word 'wave' adequately conveys the true nature of a basic quantum entity, but both provide useful partial pictures. The laws governing these sub-microscopic quantum entities differ from those governing our everyday classical world. For example, quantum particle can exist in two or more states or locations simultaneously, where such a multiple coexisting superposition of alternatives (each alternative being weighted by a complex number) would be describe mathematically by a quantum wave function. We don't see superposition in the consciously perceived world; we see objects and particles as material, classical things in specific locations and states. Another quantum property is 'non-local entanglement,' in which separated components of a system become unified, the entire collection of components being governed by one common quantum wave function. The parts remain somehow connected, even when spatially separated by significant distances (e.g. over 10 kilometers, Tittel et al., 1998). Quantum superposition of bit states (quantum bits, or qubits) can be interconnected with one another through entanglement in quantum computers. However, quantum entanglements cannot, by themselves, be used to send a message from one part of an entangled system to another; yet entanglement can be used in conjunction with classical signaling to achieve strange effects - such as the strange phenomenon referred to as 'quantum teleportation' - that classical signaling cannot achieve by itself (e.g. Bennettand Wiesner, 1992; Bennett et al., 1993; Bouwmeester et al 1997; Macikic at al., 2002).

The issue of why we don't directly perceive quantum superposition is a manifestation of the measurement problem. Put more precisely, the measurement problem is the conflict between the fundamental procedures of quantum mechanics. One of these procedures, referred to as unitary evolution, denoted here $U$, is the continuous deterministic evolution of the quantum state [i.e. of the wave function of the entire system] according to the fundamental Shrödinger equation otherwise we take the WDW equation [i.e. of the wave function $\Psi\left(R, R_{I}\right)$ over the complex space-time $R+i R_{I}$ ]. The other is the procedure that is adopted whenever a measurement of system or observation - is deemed to have taken place, where the quantum state is discontinuously and probabilistically 
replaced by another quantum state (referred to, technically, as an eigen-state or pseudo) of a mathematical operator that is taken to describe the measurement. This discontinuous jumping of the state is referred to as the reduction of the sate (or the collapse of the Shrödinger type wave function), and will be denoted here by the letter R. the conflict that is termed the measurement problem (or perhaps more accurately as the measurement paradox) arises when we consider the measuring apparatus itself as a quantum entity, which is a part of the entire quantum system consisting of the original system under observation together with this measuring apparatus. The apparatus is, after all, constructed in terms of the continuous and deterministic U. How, then, can the discontinuous and probabilistic $\mathrm{R}$ come about as a result of the interaction (measurement) between two parts of the quantum system? This is the measurement problem (or paradox). There are many ways that quantum physicists have attempted to come to terms with this conflict (see, for example, Bell 1966, Bohm 1951, Rae 1994, Polkinghorne 2002, Penrose 2004). It was shown in my previous published paper (The Complex Model Of The Quantum Universe) in which indicates the large or small matter energy density in the vapor phase (10- dimensional space-time) changes to the large or small matter energy density in the liquid phase (4- dimensional space-time) and hence it is found, an important fact, that the existence of discrete structure in the universe, ranging from galaxies to super-clusters.

In the early 20-th century, the Danish physicist Niels Bohr, together with Werner Heisenberg, proposed the pragmatic 'Copenhagen interpretation', according to which the wave function of a quantum system, evolving according to $\mathrm{U}$, is not assigned any actual physical 'reality', but is taken as basically providing the needed 'book-keeping' so that eventually probability values can be assigned to the various possible outcomes of a quantum measurement. The measuring device itself is explicitly taken to behave classically and no account is taken of the fact that the device is ultimately built from quantum-level constituents. The probabilistic are calculated, once the nature of the measuring device is known, from the state that the wave function has Uevolved to at the time of the measurement. The discontinuous 'jump' that the wave function makes upon measurement, according to R, is attributed to the change in 'knowledge' that the result of the measurement has on the observer's knowledge of the quantum system, the jumping is considered simply to reflect the jump in the observers knowledge state, rather than in the quantum system under consideration.

Many physicists remain unhappy with such a point of view, however, and regard it largely as a 'stopgap', in order that progress can be made in applying the quantum formalism, without this progress being held up by a lack of serious quantum ontology, which might provide a more complete picture of what is actually going on. One may ask in particular, what it is about a measuring device that allows one to ignore the fact that it is itself made from quantum constituents and is permitted to be treated entirely classically. A good many proponents of the Copenhagen standpoint would take the view that while the physical measuring apparatus ought actually to be treated as a quantum system, and therefore part of an over-riding wave-function evolving according to $\mathrm{U}$, it would be the conscious observer, examining the reading on that device, who actually reduces the state, according to R, there by assigning a physical reality to the particular observed alternative resulting from the measurement. Accordingly, before the intervention of the observers consciousness, the various alternatives of the result of the measurement including the different states of the measuring apparatus would, in effect, still coexist in superposition, in accordance with what would be the usual evolution according to U. In this way, the Copenhagen viewpoint puts consciousness outside science, and does not seriously address the nature and physical role of superposition nor the question of how large quantum superposition like Shrödinger's superposed live and dead cat might actually itself become one thing or another.

A more extreme variant of this approach is the 'multiple world hypothesis' of Everett (1957) in which each possibility in a superposition evolves to form its own universe, resulting in an infinite multitude of coexisting 'parallel' worlds. The stream of consciousness of the observer is supposed somehow to 'split', so that there is one in each of the worlds at least in those worlds for which the observer remains alive and conscious. Each instance of the observer's consciousness experiences a separate independent world, and is not directly aware of any of the worlds.

A more 'down-to-earth' view point is that of environmental de-coherence, in which interaction of a superposition with its environment 'erodes' quantum states, so that instead of a single wave function being used to describe the state, a more complicated entity is used, referred to as a density matrix. However de-coherence does not provide a consistent ontology for the reality of the world, in relation to the density matrix (see, for example, Penrose 2004, sections 29.3-6), and provides merely a pragmatic procedure. Moreover, it does not address the issue of how $\mathrm{R}$ might arise in isolated system, nor the nature of isolation, in which an external 'environment' would not be involved, nor does it tell us which part of a system is to be regarded as the 'environment' part, and it provides no limit to the size of that part which can remain subject to quantum superposition. 
Still other approaches include various types of objective reduction (OR) in which a specific objective threshold is proposed to cause quantum state reduction (e.g. Kibble 1981; Pearle 1989; Ghirardi et al., 1986; Percival, 1994; Ghirardi, 2011).

The quantum pioneer Erwin Shrödinger took pains to point out the difficulties that confront the Uevolution of a quantum system with his still-famous thought experiment called 'Shrödinger's cat'. Here, the fate of a cat in a box is determined by magnifying a quantum event (say the decay of a radioactive atom, within a specific time period that would provide a $50 \%$ probability of decay) to a microscopic action which would kill the cat, so that according to Shrödinger's own U-evolution the cat would be in a quantum superposition of being both dead and alive at the same time. If this U-evolution is maintained until the box is opened and the cat observed, then it would have to be the conscious human observing the cat that results in the cat becoming either dead or alive (unless, of course, the cat's own consciousness could be considered to have already served this purpose). Shrödinger intended to illustrate the absurdity of the direct applicability of the rules of quantum mechanics (including his own U-evolution) when applied at the level of a cat. Like Einstein, he regarded quantum mechanics as an in-complete theory, and his 'cat' provided an excellent example for emphasizing this incompleteness. There, is a need for something to be done about quantum mechanics irrespective of the issue of its relevance to consciousness.

\section{How consciousness arises from brain action.}

How does the brain produce consciousness? The mechanism by which the brain produces consciousness remains mysterious (Koch, 2004). The prevalent scientific view is that consciousness somehow emerges from complex computation among simple neurons which each receive and integrate synaptic inputs to a threshold for bit-like firing. The brain as network of $10^{11}$ 'integrateand-fire' neurons computing by bit-like firing and variable strength chemical synapses is the standard model for computer simulations of brain function, e.g. in the field of artificial intelligence ('AI').

The brain-as-computer view can account for non-consciousness cognitive functions including much of our mental processing and controlled behavior. Such non-conscious cognitive processes are deemed 'zombie' modes, 'auto pilot' or 'easy problems'. The 'hard problem' (Chalmers, 1996) is the question of how cognitive processes are accompanied or driven by phenomenal conscious experience and subjective feelings, referred to by philosophers as 'qualia'. Other issues also suggest the brain-as-computer view may be incomplete, and that, other approaches are required. The conventional brain-as-computer view fails to account for: 'The hard problem'. Distinctions between consciousness and non-consciousness processes are not addressed, consciousness is assumed to emerge at a critical level (neither specified testable) of computational complexity mediating otherwise non-conscious processes. 'Non-computable' thought and understanding, e.g. as shown by Godël's theorem (Penrose, 1980, 1994).Causal efficacy of consciousness and any semblance of free will. Because measurable brain activity corresponding to a stimulus often occurs after we have responded (seemingly consciously) to that stimulus, the brain as-computer view depicts consciousness as epiphenomenal illusion (Dennett, 1991; 1995; Wegner, 2002).

\section{Cognitive behaviors of single cell organisms.}

Protozoan's like Paramecium can swim, find food and mates, learn, remember and have sex, all without synaptic computation (Sherrington, 1957). In the 1980s Penrose and Hameroff (Separately) began to address these issues, each against the grain of mainstream views.

Hameroff had been intrigued by seemingly intelligent, organized activities inside cells, accomplished by protein polymers called microtubules (Hameroff and Watt, 1982; Hameroff, 1987). Major components of the cell's structural cytoskeleton, microtubules also accounted for precise separation of chromosomes in cell division, complex behavior of Paramecium, and regulation of synapses within brain neurons. He assured the intelligent function and periodic lattice structure of microtubules suggested they might function as some type of bio-molecular computer.

Microtubules are self-assembling polymers of the peanut-shaped protein dimer tubulin, each tubulin dimer (110,000 atomic mass unit) being composed of an alpha and beta monomer. Thirteen linear tubulin chains ('proto-filaments') align side-to-side to form hollow microtubule cylinders (25 nanometers diameter) with two types of hexagonal lattices. The A-lattice has multiple winding patterns which intersect proto-filaments at specific intervals matching the Fibonacci series found widely in nature and hence possessing a helical symmetry, which suggestively to a large-scale quantum process. 


\section{Objective Reduction.}

In 1989 Penrose published 'The Emperor's New Mind', which was followed in 1994 by 'Shadow of the Mind'. Critical of AI, both books argued by appealing to Gödel's theorem and other considerations, that certain aspects of human consciousness, such as 'understanding' must be beyond the scope of any computational system, i.e. 'non-computable'. Non-computability is a perfectly well-defined mathematical concept, but it had not previously been con sidered as a serious possibility for the result of physical actions. The non-computable ingredient required for human consciousness and understanding, Penrose suggested, would have to lie in an area where our current physical theories are fundamentally incomplete, though of important relevance to the scales that are pertinent to the operation of our brains. The only serious possibility was the incompleteness of quantum theory- an incompleteness that both Einstein and Shrödinger had recognized, despite quantum theory having frequently been argues to represent the pinnacle of $20^{\text {th }}$ century scientific achievement. This incompleteness is the unresolved issue referred to as the 'measurement problem', which we consider in more detail in the present dissertation. One way to resolve it would be to provide an extension of the standard framework of quantum mechanics by introducing a Generalized Gaussian Energy Group (GGEG) and Penrose introduced an objective form of quantum state reduction, termed 'OR', (objective reduction). In Penrose (1989), the tentatively suggested OR proposal would have its onset determined by a condition referred to there as "the one-graviton criterion'. However, in Penrose (1995), a much better-founded criterion was used, now sometimes referred to as the Dio'si - Penrose proposal (henceforth 'DP'; Dio'si 1987, 1989, Penrose 1993, 1996, 2000, 2009). This is an objective physical threshold, providing a plausible lifetime for quantum superposed states. Other such OR proposals had also been put forward, from time to time (e.g. Kibble 1981, Pearle 1989, Pearle and Squires 1994, Ghirardi et al., 1986, 1990; Ghirardi 2011) as solutions to the measurement problem, but had not originally been suggested as having anything do with the consciousness issue.

\section{Goldstone's Theorem with Symmetry Breaking:}

The concept of broken symmetry is clarified by reflecting on the process by which a crystal may be formed. Suppose the crystal to be solid carbon-dioxide ("dry ice"), and suppose the carbon-dioxide initially to be in the gaseous state, contained in some relatively large vessel ("infinite in size"). The gas is slowly cooled. At the temperature of the gas-solid phase transition a crystalline nucleus forms at some point in the gas (which may be compared with the different stages of the very early universe from infinity of the gas-condensed gas phase transition upto vapor-solid nucleus forms). The nucleus thereafter grows until the gas pressure falls to that on the gas-solid coexistence curve (i.e. to the vapor pressure of the solid). From the point of view of symmetry the condensation is a quite remarkable development. In the "infinite" gas the system of the universe is symmetric under a continuous translation until the energy group SU(11), but the condensed solid (as our universe) has a lower symmetry (constructed space-time as different as before)! It is invariant only under a discrete translation group. Furthermore the location of the crystal is arbitrary, determine by the accident of the first microscopic nucleation. In that nucleation process the symmetry of the system suddenly and spontaneously lowers, and it does so by a non-predictable, random event. The symmetry of the system is "broken".

Macroscopic sciences, such as solid state physics or thermodynamics, are qualitatively different form "microscopic" sciences because of the effects of broken symmetry, as was pointed out by P.W. Anderson in an early but profound and easily readable essay.

At sufficiently high temperature systems always exhibit the full symmetry of the "mechanical potential" (i.e., of the Lagrangian or Hamiltonian functions). There do exist permissible micro-states with lower symmetry, but these states are grouped in sets which collectively [for example, SU(11)] exhibit the full symmetry. Thus the microstates of a gas do include states with crystal-like spacing of the molecules- in fact, among the microstates all manner of different crystal-like spacing are represented, so that collectively the states of the gas retain no overall crystalline whatever. However, as the temperature of the gas is lowered the molecules select that particular crystalline spacing of lowest energy, and the gas condenses into the corresponding crystal structure. This is a partial breaking of the symmetry. Even among the microstates with this crystalline periodicity there are a continuum of possibilities available to the system, for the incipient crystal could crystalline with any arbitrary position. Given one possible crystal position there exist infinitely many equally possible positions, slightly displaced by an arbitrary function of a "lattice constant". Among these possibilities, all of equal energy, the system chooses one position (i.e., a nucleation center for the condensing crystallite) arbitrarily and "accidentally". An important general consequence of broken symmetry is formulated in the Goldstone theorem. It asserts that any system with broken symmetry (and with certain weak restrictions on the atomic interactions) has a spectrum of excitations for which the frequency approaches zero as the wave length becomes infinitely large. 
So for the crystal discussed here the Goldstone theorem ensures that a phonon excitation spectrum exists, and that its frequency vanishes in the long wave length limit.

In summary, then, the volume emerges as a thermodynamic co-ordinate by virtue of a fundamental symmetry principle grounded in the concept of broken symmetry.

\section{Thermodynamics with gravity:}

An astronomers, however, have long had to contend with the conjunction of thermodynamics and gravitation. In the broadest sense, thermodynamics regulates the organization of activity in our universe, and gravity controls the dynamics, at least on the large scale.

One of the central difficulties about the thermodynamics of gravitating systems is the apparent absence of true equilibrium. This problem, which can lead directly to 'peculiar' effects, has long been known to astronomers. Stars are hot, self-gravitating balls of gas inside which the weight of the star is supported by its own internal kinetic or zero-point quantum presence. Unlike ordinary laboratory thermodynamic systems, a star is made hotter, not by adding energy, but by removing it. We consider the change takes place by the latent energy group SU(6) into the matter energy group of SU(5) of the super unified group SU(11) and vice-versa. So, initially heat can be found from internal energy of the thermo-statistical particles.

\section{Creation of Black-Holes:}

The key to understanding black-holes, and especially their connection with thermodynamics, is to appreciate the meaning of the so-called event horizon. Consider a very compact and massive star. The strength of gravity [due to the excess production of $\mathbf{J}_{\mathrm{k}}$ bosons of $\mathrm{SU}(6)$ in the very early universe] at its surface can be increased by the decomposition of the energy group SU(11) from another phase by the phase transition system. In the very early universe the production of $\mathrm{J}_{\mathrm{k}}$ bosons are much more and hence more bosons of the matter group $\mathrm{SU}(5)$ were increased by changing the energy particles of the group $\mathrm{SU}(6)$. According to our assumption, our universe started from the 10-dimensional super-gravity in the large scale and there is a flatness with the compactification of extra-dimension up-to 7-dimensional universe. According to the Einstein's 4-dimensional universe, gravity affects the properties of light, and this is manifestation in the behavior of light rays which leave the surface of the star travelling radially outwards. Because the light has to 'do work' to overcome the surface gravity and escape from the star, its energy, and hence frequency, will be some-what diminished. This famous gravitational red-shift has been measured in light leaving such relatively low gravity objects as the Sun, and the Earth. For more compact and massive objects the red-shift can become enormous. Even on Newtonian grounds it is clear (as pointed out by Peirre Laplace as long as 1798) that when the escape velocity from the surface of a star exceeds that of light, something odd must happen. According to both Newtonian gravity and Einstein's relativity, this turn of events comes about for a spherical, uncharged star if the radius of the star shrinks below $2 \mathrm{GM} / \mathrm{c}^{2}$, where $\mathrm{M}$ is the mass, $\mathrm{c}$ is the speed of light, and $\mathrm{G}$ is the Newtonian gravitational constants. This size is very small, being of the order of $1 \mathrm{~km}$ for the Sun and $1 \mathrm{~cm}$ for the Earth. A straightforward calculation shows that as the star approaches the critical radius, the light from its surface becomes redshifted without limit, so that it can no longer been seen; it is black.

A useful picture of the event horizon can be obtained by imaging spherical wave-fronts of light which are emitted radially outwards from different surfaces $r=$ constants. Those spheres travelling from $r>2 \mathrm{GM} / \mathrm{c}^{2}$ gradually expands and eventually escape to infinity, but those emitted inside the critical radius actually shrink towards the centre, even though they are emitted in a direction away from the centre. Crudely speaking, the gravity there is so strong that it drags the light backwards. The event horizon is the spherical surface of light that just escapes to infinity after an infinite duration.

According to relativity, matter and information cannot propagate faster than light, so if light can- not escape from inside the horizon, neither can anything else. Thus, once the star has retreated through this surface it can never return to the outside universe.

The region inside the horizon, once the star has shrunk away to nothing, i.e. in another phase, is empty (so called) and from the exterior universe i.e. from the present phase, black and inaccessible. It is therefore called a black-hole. Astronomers widely believe that black holes will form as the natural end state of the evolution of massive stars, but so far there is no direct observational evidence of their existence. The most general known solutions to Einstein's field equations of general relativity which contain black holes are the socalled Kerr-Newman family, which describe an asymmetric, matter-free space-time representing a black-hole which rotates and carries an electric charge. These solutions form a three-parameter set, labeled by the total mass-energy ' $\mathrm{M}$ ', the angular momentum ' $\mathrm{J}$ ' $=|\mathrm{J}|$ and the electric charge ' $\mathrm{e}$ '. For $\mathrm{J}=0$, suggests that flatness occurred from 10-dimensional to 7-dimensional universe and then from 7-dimensional to 4-dimensional that black-holes have a very high entropy, and represent in some sense the maximum entropy, equilibrium end state of gravitational collapse. 


\section{The Laws of Classical Black-Hole 'Thermodynamics':}

There is a general tendency for self-gravitating systems to grow rather than shrink because gravity always attracts. The behavior between the black-holes and thermodynamic equilibrium systems were noted some time ago (for a review see Carter 1973). In the black-hole case, the inability for light to change from inside the event horizon precludes the escape of any material, so the horizon acts as a sort of asymmetric one-way surface: things can fall in and make the hole bigger but not come out and make it smaller [i.e. slowly changes to another phase]. This is reminiscent of the second law of thermodynamics, in which there is an asymmetric tendency for a one-way increase in entropy. The size of the black-hole is analogous to the entropy. The above statement can be explained as, in the theory of SU(11), it is possible to change any of 30-bosons of SU(5) of the matter energy group into any of 30-bosons of SU(6) of the latent energy group and vice-versa by the exchange of J-bosons of SU(11).

The analogy is almost trivial for a spherical, electrically neutral (Schwarzschild) black-hole. In the more general case of black-holes that possess angular momentum ' $\mathrm{J}$ ' by SU(6) and electric charge ' $\mathrm{e}$ ' by $\mathrm{U}(1)$, the size of the black hole depends both on ' $\mathrm{J}$ ' and ' $\mathrm{e}$ ' in a rather complicated way. If the total surface area of the horizon is used as a measure of size then this is given by the formula (Samrr 1973):

$$
A=4 \pi\left[2 M^{2}-e^{2}+2 M^{2}\left(1-\frac{e^{2}}{M^{2}}-\frac{J^{2}}{M^{4}}\right)^{\frac{1}{2}}\right] \quad\left[\text { Where, } M^{2}=e^{2}+\frac{e^{4}}{4}+1\right]
$$

Where $\mathrm{e}^{2}<\mathrm{M}^{2}$ and $\mathrm{J}^{2}<\mathrm{M}^{4}$ (throughout, units with $\mathrm{G}=\mathrm{c}=1$ will be used) so it is clear at a glance whether a disturbance to the black-hole which changes both 'e' and ' $J$ ', as well as ' $\mathrm{M}$ ', will always increase the total area.

An example due to Penrose (1969) concerns a method for extracting mass-energy from a rotating black-hole. The mechanism consists of propelling a small body into the region just outside the event horizon where (due to dragging effect on the space surrounding the black-hole caused by its rotation) some particle trajectories possess negative energy relative to infinity. Due to the symmetry breaking of SU(11), we get mainly two types of energy source as $\mathrm{SU}(6)$ and $\mathrm{SU}(5)$, one of which i.e. particles of the energy group SU(6) is placed on one of these negative energy paths, and this part changes to $\mathrm{SU}(5)$, so reduce the total mass $\mathrm{M}$ of the 'hole' somewhat and hence the mass-energy of SU(5) thereby released by this sacrificed particles of the latent energy group SU(6) which is ejected to infinity at high speed. During this energy transfer the black-hole's rotation rate is diminished somewhat, so $\mathrm{J}$ also decreases. Thus, when $\mathrm{J}$ decreases, the area $\mathrm{A}$ increases, but when $\mathrm{M}$ decreases, the area decreases. The change in $\mathrm{M}$ and $\mathrm{J}$ are therefore in competition, but a careful calculation shows that $\mathrm{J}$ always wins and the area increases.

Actually, if the class of all trajectories is studied, it is found that in general the area increases by an amount corresponding to a considerable fraction of mass energy. However, the efficiency of energy extraction can be improved by approaching closely to a limiting class of trajectories for which the event horizon area remains constant. The limiting case is therefore reversible and corresponds to an isentropic change in thermodynamics. In practice, $100 \%$ efficiency (complete reversibility) would be impossible.

This strong analogy between event horizon area and entropy led to use of the name 'second law' in connection with Hawking's area theorem, which is therefore written as: $\mathrm{dA} \geq 0$, [equality corresponding to reversibility] There are also analogous of the Zeroth, first and third laws of thermodynamics. From the equation,

$$
\mathrm{r}=\mathrm{r}_{ \pm} \equiv \frac{1}{2} \mathrm{M} \pm \frac{1}{2}\left(\mathrm{M}^{2}-2 \mathrm{e}^{2}\right)^{1 / 2}
$$

[These are not singularities in the geometry itself but in the co-ordinate system (t, r), similar to these which occur to latitude and longitude on the surface of a sphere at the poles. In fact, the outer surface $r_{+}$corresponds to the event horizon: notice that $r_{+} \rightarrow M$ as e $\rightarrow 0$. This surface has global significance, but locally an internal observer would find nothing unusual about the space-time geometry there. The inner surface $\mathrm{r}_{-}$is another type of horizon inside the hole- itself.] [See, 'The Complex Quantum-State of Black-Hole and Thermostatistics' (IOSR-JM: e-ISSN: 2278-3008, p-ISSN: 2319-7676. Volume 8, Issue 5 (Nov. - Dec. 2013), PP 01-19)].

Thus, we can obtain, $\quad \mathrm{dM}=(8 \pi)^{-1} \mathrm{kdA}+\Omega \mathrm{dJ}+\phi$ de

Where $(8 \pi)^{-1} \mathrm{k} \equiv \frac{\partial \mathrm{M}}{\partial \mathrm{A}}$,etc. which is really just an expression of mass-energy conservation and corresponds to the first law. If A plays the role of entropy then we see from the above equation, that $\mathrm{k}$ plays the role of temperature (kdA $\sim \mathrm{TdS})$. The interesting thing is that $\mathrm{k}$ can be shown to be constant across the event horizon surface. We thus have an expression of a 'Zeroth' law, analogous to the thermodynamic one of which says that in thermodynamic equilibrium there exists a common temperature parameter for the whole system. The quantity $\mathrm{k}$ is known as the surface gravity of the black-hole. Its significance lies in the fact that it determines the e-folding time which controls the rate at which the collapsing star red-shifts and approaches equilibrium. For a Schwarzschild hole $\mathrm{k}=(4 \mathrm{M})^{-1}$ and the constant $8 \pi$ in the above equation has been chosen to agree with this. The remaining terms in the above equation simply describe the work done (energy extracted) from changes in 
angular momentum $(\Omega \mathrm{d})$ ) and electric charge ( $\phi \mathrm{de})$ and have a very obvious structure: $\Omega$ is the (magnitude of) angular velocity and $\boldsymbol{\phi}$ the electric potential at the event horizon.

Finally, there is the third law. It is straightforward to show that if $\mathrm{J}^{2}$ or $\mathrm{e}^{2}$ become large enough such that:

$$
\frac{\mathrm{J}^{2}}{\mathrm{M}^{4}}+\frac{\mathrm{e}^{2}}{\mathrm{M}^{2}}=1 \quad \text { i. e. } \quad \mathrm{J}^{2}=\left(\frac{\mathrm{e}^{4}}{4}+1\right)\left(\mathrm{e}^{2}+\frac{\mathrm{e}^{4}}{4}+1\right)
$$

then $\mathrm{k}$ vanishes (although A does not). This corresponds to absolute zero (though with finite entropy). A blackhole with parameters given by the above equation is known as an extreme Kerr-Newman black-hole. It is the limiting case of an object which still possesses an event horizon. Should the left-hand side become even infinitesimally greater than one, then the horizon would disappear and we would left with a naked singularity, i.e. the singularity would no longer be invisible inside a black-hole but would be able to influence, and be observed by, the outside universe. This circumstance is considered so undesirable for physics that most physicists believe in the so-called cosmic censorship hypothesis due to Penrose (1969): naked singularities cannot form from gravitational collapse. Cosmic censorship implies the un-attainability of 'absolute zero', $\mathrm{k}=0$, so it plays the role of the third law.

\section{The conceptual Foundations of Thermo-statistics:}

Thermo-statistics characterizes the equilibrium state of microscopic system without reference either to the specific forces or to the laws of mechanical response. Instead thermo-statistics characterizes the equilibrium state as the state that maximizes the disorder, a quantity associated with a conceptual framework ("Information Theory") outside of conventional physical theory. The question arises as to whether the postulator basis of thermo-statistics thereby introduces new principles not contained in mechanics, electromagnetism, and the like or whether it borrows principles in unrecognized form from that standard body of physical theory. In either case, what are the implicit principles upon which thermo-statistics rests?

There are, in my view, two essential basis underlying thermo-statistical theory. One rooted in the statistical properties of large complex systems. The second rests in the set of symmetries of the fundamental laws of physics. The statistical feature veils the incoherent complexity of the atomic dynamics, thereby revealing the coherent effects of the underlying physical symmetries. The relevance of the statistical properties of large complex systems is universally accepted and reasonable evident. The essential property is epitomized in the "central limit theorem". Which states (roughly) that the probability density of a variable assumes the "Gaussian" form if the variable is itself the resultant of a large number of independent additive sub-variables. Although one might naively hope that measurements of thermo-dynamic fluctuation amplitudes could yield detailed information as to the atomic structure of a system, the central limit theorem precludes such a possibility. It is this insensitivity to specific structural or mechanical detail that underlies the universality and simplicity of thermo-statistics.

\section{Energy Conservation Principle:}

The development of the principle of conservation of energy has been one of the most significant achievements in the evolution of physics. The first recognition of a conservation principle, by Leibniz in 1693 when Leibniz observed that $\frac{1}{2} \mathrm{mv}^{2}+\mathrm{mgh}$ is a conserved quantity for a mass particle in the earth's gravitational field. As successively more complex systems were studied it was found that additional terms had to be appended to maintain a conservation principle, but that in each case such an ad-hoc addition was possible. The development of electro-magnetic theory introduced the potential energy of the interaction of electric charges, subsequently to be augmented by the electromagnetic field energy. In 1905 Albert Einstein was inspired to alter the expression for the mechanical kinetic energy, and even to associate energy with stationary mass, in order to maintain the principle of energy conservation. In the 1930 Enrico Fermi postulated the existence of the neutrino solely for the purpose of retaining the energy conservation law in nuclear reaction. And so the process continues, successively accreting additional terms to the abstract concept of energy, which is defined by its conservation law. That conservation law was evolved historically by a long series of successive rediscoveries. It was now based on the assumption of a symmetry of the new type of energy source $\mathrm{SU}(6)$ [as well as $\mathrm{SU}(12)$; $\mathrm{SU}(24) ; \ldots . . .$. etc.] by the time translation system $(\mathrm{t} \rightarrow \mathrm{t}+$ constant $)$. Which was discussed elaborately in my previous published paper (see, NK Bhadra; The Complex Model of the Universe; IOSR-JM; 2, 4, pp-41), where we consider that our universe [actually started the journey from infinite space-time i.e. from Bip-Rip singularity with the symmetry breaking of $\mathrm{SU}(23)$; $\mathrm{SU}(47) ;$.....etc.] started from 10-dimensional space-time instead of 4dimensional Einstein's universe with the symmetry breaking of the special unitary group SU(11) from 7dimensional flat space-time, there exists two types of energy sources of the Gaussian energy group SU(6) and $\mathrm{SU}(5)$, i.e. it was considered $\mathrm{SU}(6)$, a new type of energy source called latent energy group, so the conservation law of energy maintain due to the addition of SU(6) with SU(5) etc. for our universe and hence the space time is actually shifted as $t \rightarrow t_{0}$. So it is conventional therefore to adopt some particular state of a system as a fiducial state, the energy of which [i.e. SU(11)] is arbitrarily taken as zero. The energy of a system in any other state, 
relative to the energy of the system in the fiducial state, is then called the thermodynamic internal energy of the system in that state and is denoted by the symbol W. So for thermodynamic equilibrium there exists particular states of simple system that, macroscopically, are characterized completely by internal energy $\mathrm{W}$, the volume $\mathrm{V}$, and the mole numbers $\mathrm{N}_{1}, \mathrm{~N}_{2}, \mathrm{~N}_{3}, \ldots \ldots \ldots \mathrm{N}_{\mathrm{r} \text {. }}$ of the chemical components.

A physical consequence of symmetry is formulated in "Noethers's theorem". The theorem asserts that every continuous symmetry of the dynamical behavior of a system (i.e. of the dynamical equation and the mechanical potential) implies a conservation law for that system.

The dynamical equation for the motion of the centre of mass point of any material system is Newton's law. If the external force does not depend upon the co-ordinate $\mathrm{x}$, then both the potential and the dynamical equation are symmetric under spatial translation parallel to the $\mathrm{x}$-axis. The quantity that is conserved as a consequence of this symmetry is the x-component of the momentum. Similarly the symmetry under translation along the $y$ or $\mathrm{z}$ axes results in the conservation of the $\mathrm{y}$ or $\mathrm{z}$ components of the momentum. Symmetry under rotation around the $\mathrm{z}$-axis implies conservation of the $\mathrm{z}$-component of the angular momentum.

Of enormous significance for thermo-statistics is the symmetry of dynamical laws under time translation. That is, the fundamental dynamical laws of physics (such as Newton's law, Maxwell's equations, and Schrödinger's equation) are unchanged by the transformation $t \rightarrow t^{\prime}+t_{0}$ (i.e., by a shift of the origin of the scale of time). If the external potential is independent of time, Noether's theorem predicts the existence of a conserved quantity. That conserved quantity is called the energy. The evolution of the energy concept for macroscopic thermodynamic systems was even more difficult. The pioneers of the subject were guided neither by a general a priori conservation theorem nor by any specific analytic formula for the energy. Even empiricism was thwarted by the absence of a method of direct measurement of heat transfer. Only inspired in-sight guided by faith in the simplicity of nature somehow revealed the interplay of the concepts of energy and entropy, even in the absence of a priori definitions or of a means of measuring either.

\section{The Generalized "First law" of thermodynamics:}

In accepting the existence of a conserved macroscopic energy function as the first postulate of thermodynamics, we anchor that postulate directly in Noether's theorem and the time-translation symmetry of physical laws. There are seven "first integrals of the motion" (as the conserved quantities are known in mechanics). These seven conserved quantities, all arises as Lagrange parameters and they play completely symmetric roles in the theory of the energy, the three components of linear momentums and the three components of the angular momentum; and they follow in parallel fashion from the translation in "space-time" and from rotation. In fact, the energy is not unique in thermo-statistics. The linear momentum and angular momentum play precisely parallel roles. The asymmetry in our account of thermo-statistics is a purely conventional one that obscures the true nature of the subject. The proper "the first law of thermo-dynamics", (or the first postulate in our formulation) is the symmetry of the laws of physics under space-time translation and rotation, and consequent existence of conserved energy, momentum, and angular momentum functions.

\section{Generalized Special Unitary Group:}

For a field F, the generalized special unitary group over F, SU(s, p, F) is the group of all linear transformations of determinant 1 of a vector-space of rank $n=p+s$ over $F$ which leave invariant a nondegenerate, Hermitian form of signature $(s, p)$. This group is often referred to as the special unitary group of signature $(s, p)$ over $F$. The field $F$ can be replaced by a commutative ring in which case the vector-space is replaced by a free module.

In particular for fix Hermitian matrix $H$ of signature $(s, p)$ in $G L(n, R)$, all $M € S U(s, p, R)$ satisfy $M^{*} \mathrm{HM}=\mathrm{H}$, with det. $\mathrm{M}=1$. The notation $\mathrm{SU}_{\mathrm{s}, \mathrm{p}}$ without reference to a ring or field, in this case is referred to $\mathrm{A}$ and this gives one of the classical lie groups. The standard choice for $\mathrm{H}$ when $\mathrm{F}=\mathrm{A}$ is

$$
\mathrm{H}=\left(\begin{array}{ccc}
0 & 0 & \mathrm{i} \\
0 & \mathrm{I}_{\mathrm{n}-2} & 0 \\
-\mathrm{i} & 0 & 0
\end{array}\right)
$$

However, there may be better choices for $\mathrm{H}$ for certain dimensions which exhibit more behavior under restriction to subrings of $\mathrm{A}$.

\section{Important Subgroup:}

In the theory of Gauge transformation in physics, the special unitary group is used to represent Bosonic symmetries. In the theories of symmetry breaking, it is important to find the subgroups of the special unitary. Important subgroups of SU(n) that are important in GUT physics (also in the present dissertation) super unified theory are, for $\mathrm{p}>1, \mathrm{n}-\mathrm{p}>1$, with $\mathrm{SU}(\mathrm{n}) \supset \mathrm{SU}(\mathrm{p}) \times \mathrm{SU}(\mathrm{n}-\mathrm{p}) \times \mathrm{U}(1)$. For completeness there are also the orthogonal sympletic subgroup: $\quad \mathrm{SU}(n) \supset \mathrm{O}(\mathrm{n})$; $\mathrm{SU}(2 \mathrm{n}) \supset \mathrm{US} p(2 n)$. Since the rank of $\mathrm{SU}(\mathrm{n})$ is $n-1$ and $U(1)$ 
is 1 , a useful check is that the sum of the ranks of the subgroups is less than or equal to the rank of original group $\mathrm{SU}(\mathrm{n})$ which is a subgroup of various other lie group : $\mathrm{SO}(2 \mathrm{n}) \supset \mathrm{SU}(\mathrm{n})$; US $\mathrm{p}(2 \mathrm{n}) \supset \mathrm{SU}(\mathrm{n})$; $\operatorname{spin}(4)=$ $\mathrm{SU}(2) \times \mathrm{SU}(2)$. Finally it can be mention that $\mathrm{SU}(2)$ is the double covering group of $\mathrm{SO}(3)$, a relation that plays an important role in the theory of rotations of 2- spinors in non-relativistic quantum mechanics.

\section{Appearance of the Universe:}

There is no consensus yet on how the universe initially came to be, the general assumption is that perhaps an energetic fluctuation caused the universe to tunnel into the existence from quantum foam. The question of why the large energy of the universe is in a dark, i.e. not found in practical, the observed vacuum energy is so small in comparison to the scales of particle physics is known as cosmological constant problem. It is generally thought to be easier to imagine an unknown mechanism which would set vacuum parameter exactly to zero and so it can be considered that there exists several unification from the very early universe. This class of symmetry group can be expressed mathematically as $\mathrm{SU}(11) \supset \mathrm{SU}(5) \times \mathrm{SU}(6) \times \mathrm{U}(1)$; $\mathrm{SU}(23) \supset \mathrm{SU}(12) \times$ $\mathrm{SU}(11) \times \mathrm{U}(1) ; \mathrm{SU}(47) \supset \mathrm{SU}(24) \times \mathrm{SU}(23) \times \mathrm{U}(1) ; \ldots \ldots \ldots . .$. so on. We can assume $\mathrm{SU}(5)$ as matter energy group and SU(6) a new type of energy sources may be called latent energy group which changes the matter energy group into $\mathrm{SU}(11)$ [similarly, apply for $\mathrm{SU}(23)$; $\mathrm{SU}(47)$ etc.], i.e. to the super unified theory . So, it is considered that the breakdown of SUT(super unified theory) symmetry group SU(11), breaks into fundamental group SU (5) $\times \mathrm{SU}(6)$ leads to a phase transition and then larger fundamental group $\mathrm{SU}(5)$ which also breaks into subgroups $\mathrm{SU}(3) \times \mathrm{SU}(2) \times \mathrm{U}(1)$, in which the scalar field $\Phi$ changes. The original vacuum, i.e. false vacuum $(\Phi=\sigma)$ is no longer the true vacuum $(\Phi=0)$. The inflationary stage arises, however, if the true vacuum is not immediately attained.

It can explain how the universe appears from nothing. It seems our universe comes from nothing to the universe belongs to the energy group $\mathrm{SU}(11)$, a special unitary group having $11 \times 11$ matrices, such that

$$
\mathrm{SU}(11) \supset \mathrm{SU}(5) \times \mathrm{SU}(6) \times \mathrm{U}(1) \text {. }
$$

Thus, we have from SU(11), the Hermitian matrix H has 120 arbitrary constants. Which correspond to 120 bosons that now mediate between the different basic entities. Of these we already have 60-bosons from the matter energy group SU(5), latent energy group SU(6) and from U(1). Thus, $120-(24+35+1)=60$ more bosons are namely J-bosons are expected to link the participants of $\mathrm{SU}(6)$ with $\mathrm{SU}(5)$. Therefore, in the theory of SU(11), it is possible to change any of 30(thirty) latent energy bosons of SU(6) into any of the 30(thirty) matter energy bosons of $\mathrm{SU}(5)$ or vice-versa by the exchange of the J-boson. That is why it becomes possible to create or destroy matter particles and hence the universe seems to appear from nothing. In the present dissertation there is neither any starting point nor any ending point of the wider (measurable in quantum cosmology) universe (i.e. complex space-time). Only there exists the initial and end conditions for narrower (measurable classically) universe (i.e. Einstein space-time) which emerged from wider universe by the process of changing phase, where it is a continuous process.

Again, on the other-hand, the idea that our 4-dimensional universe might have emerged from a higher dimensional space-time is now receiving much attention where the compactification of higher dimensions plays a key role. However, the question arises of how and why this compactification occurs. From string theory we know that the compactification may take place provided that the higher dimensional manifold admits special properties, namely if the geometry of the manifold allows, for example, the existence of a suitable killing vector. However, it is difficult to understand why such manifolds are preferred and whether other possible mechanism for compactification do exist. In cosmology, on the other-hand, different kinds of compactification could be considered. For example, in an approach, called dynamical compactification, the extra-dimensions evolve in time towards very small sizes and the extra dimensional universe reduces to an effective 4-dimensional one. This type of compactification was considered in my previous published paper ['The complex model of the universe' of IOSR-JM, vol.2, 4 (2012), pp-41] with the help of Modern Kaluza-Klein theories. It is then a natural question that how an effective four dimensional universe evolve in time and whether the resulting cosmology is similar to the standard FRW four dimensional universe without extra dimensions. A universe may based on the considerations of dark energy sector. One may start from a fundamental theory including both gravity and standard model of particle physics. In this regard it is interesting to begin with $10(=4+6)$ dimensional space-time, in which case one needs a compactification of 10-dimensional super gravity theory where an effective 4-dimensional undergoes acceleration. However, it has been known for sometime that it is difficult to derive such a cosmology and has been considered that there is a no-go theorem that excludes such a possibility, if one takes the internal space to be time-independent and compact without boundary. However, it has recently been shown that one may avoid this no-go theorem by giving up the condition of timeindependence of the internal space, and a solution of the vacuum Einstein equations with compact hyperbolic internal space has been proposed based on this model. Similar accelerating cosmologies can also obtain for SM2 and SD2 branes, not only for hyperbolic but also for flat internal space. 
On the other-hand, from cosmological point of view it is not so difficult to find cosmological models in which the 4-dimensional universe undergoes an accelerating expansion and the internal space contracts with time, exhibiting the dynamical compactification. In my paper we consider the internal space as imaginary (pseudo- space, $b=i R$ ), where the matter belongs to another phase by the phase transition process with the help of the latent group SU(6).

It is shown that using a more general metric and introducing matter without specifying its nature, the size of compact space evolves as an inverse power of the radius of the universe. The FRW equations of the standard four dimensional cosmology is obtained using an effective pressures expressed in-terms of the components of the higher dimensional energy-momentum tensor and the negative value of this pressure may explain the acceleration of our present universe.

One of the recent important cosmological observation is the accelerated expansion of the universe. It is known from the recent distance measurement of type Ia supernova suggest strongly an accelerating universe. This accelerating expansion is generally believed to be driven by an energy source called dark energy which provides negative pressure, such as a positive cosmological constant, or a slowly evolving real scalar field called quintessence. Moreover, the basic conclusion from all previous observations that 70 percent of the energy density of the universe is in a dark energy sector, has been confirmed after the recent WMAP. That means the matter content of the universe is composed of 70 percent of an un-clustered component of negative pressure (dark energy) and 30 percent of a clustered component of zero pressure (cold dark matter). The traditional candidate of dark energy are the cosmological constant, $\Lambda$ and quintessence. But, these approaches have some serious drawbacks from fundamental point of view. In fact, the observed energy density of the cosmological constant has a discrepancy of about 120 orders of magnitude with the theoretically predicted value from field theory models.

In the present dissertation, we consider in the very early universe up-to the Big-Rip singularity, there were several unifications. This class of symmetry group can be expressed mathematically as follows:

$$
\begin{aligned}
& \mathrm{SU}(11) \supset \mathrm{SU}(6) \times \mathrm{SU}(5) \times \mathrm{U}(1) \\
& \mathrm{SU}(23) \supset \mathrm{SU}(12) \times \mathrm{SU}(11) \times \mathrm{U}(1) \\
& \mathrm{SU}(47) \supset \mathrm{SU}(24) \times \mathrm{SU}(23) \times \mathrm{U}(1) \\
& \ldots \ldots \ldots \ldots \ldots \ldots \ldots \ldots \ldots \ldots \ldots \ldots . \ldots . \ldots . \ldots . \ldots . \ldots . \ldots . \ldots
\end{aligned}
$$

Thus we have, $\quad \mathrm{SU}(12 \mathrm{n}-1) \supset \mathrm{SU}(6 \mathrm{n}) \times \mathrm{SU}(6 \mathrm{n}-1) \times \mathrm{U}(1), \quad$ where, $\quad \mathrm{n}=2^{0}, 2^{1}, 2^{2}, 2^{3}, 2^{4} \ldots \ldots \ldots \ldots \ldots \ldots$. i.e. $\quad \mathrm{SU}(12 \mathrm{n}-1) \supset \mathrm{SU}(6 \mathrm{n}) \times \ldots \ldots \ldots \ldots \ldots \ldots \ldots \ldots \mathrm{SU}(24) \times \mathrm{SU}(12) \times \mathrm{SU}(6) \times \mathrm{SU}(3) \times \mathrm{SU}(2) \times \mathrm{U}(1)$. Hence, according to the law of symmetry breaking of the energy group, i.e. according to the symmetry breaking of the Gaussian energy group, there exists neither any Big-Bang nor any Big-Crunch singularity, only there BigRip singularity and Big-Break singularity.

All the dark energies $\mathrm{SU}(12), \mathrm{SU}(24), \ldots \ldots$ etc. are responsible for the various consciousness and intelligence of the living cells or bodies according to the progress for biological revolution as such the progress of the material universe depends on the sub-energy groups of SU (5) $\supset \mathrm{SU}(3) \times \mathrm{SU}(2) \times U$ (1) stated by the standard model of physics. Again the activities of the energy groups for consciousness are restricted to unfold for each galaxy of the universe as well as for every solar system of the galaxies. Our matter universe, i.e. quantum particle energy universe starts from SU(11), i.e. classically starts from 10-dimensional space-time. The quantum wave energy-universe actually starts from Big-Rip singularity, which was shown, that there exists pressure and densities of the energy simultaneously. Most probably, 28\% of the total energy (measuring by WMAP) were found for the symmetry breaking of the energy group SU (11) and rest $72 \%$ of the energy belongs to another phase (according to the classical mathematics which are folded) starting from Big-Rip singularity due to symmetry breaking of SU(23), SU(47), .......

\section{The cosmological model with a scalar field potential:}

Let us consider a flat Friedmann-Lemaitre-Robertson-Walker universe with metric,

With the equation of state

$$
d S^{2}=N^{2}(t) d t^{2}-a^{2}(t) d t^{2}
$$

$$
p=\gamma \rho+\frac{A}{\rho^{\alpha}} \text { (where } \gamma, \mathrm{A}, \alpha \text { are arbitrarily constants). }
$$

Where "dot" means the derivative with respect to the cosmic time t. The energy conservation condition is

Putting the value of $\mathrm{p}$, we have

$$
\dot{\rho}=-3 H(\rho+p)
$$




$$
\begin{gathered}
\dot{\rho}=-3 \rho^{1 / 2}\left(\gamma \rho+\frac{A}{\rho^{\alpha}}+\rho\right)=-3 A \rho^{\frac{1}{2}-\alpha}-3(\gamma+1) \rho^{3 / 2} \\
\text { i.e } \frac{H^{2 \alpha+1} d H}{A+(\gamma+1) H^{2 \alpha+2}}=-\frac{3 \dot{R}}{2 R} d t
\end{gathered}
$$

Integrating, we have

$$
\rho(R)=\frac{1}{(\gamma+1)^{1 /(\alpha+1)}}\left[\left(\frac{B}{R}\right)^{3(\gamma+1)(\alpha+1)}-A\right]^{\frac{1}{\alpha+1}}
$$

Again, the Friedmann equation is

$$
H^{2}=\rho
$$

Where the Hubble parameter $\mathrm{H}$ is as usual

$$
\begin{aligned}
& H \equiv \frac{\dot{R}}{R} \quad \text { i.e. } \frac{\dot{R}^{2}}{R^{2}}=\frac{1}{(\gamma+1)^{1 /(\alpha+1)}}\left[\left(\frac{B}{R}\right)^{3(\gamma+1)(\alpha+1)}-A\right]^{\frac{1}{\alpha+1}} \\
& \text { i.e. } \quad \dot{R}=\frac{1}{(\gamma+1)^{\frac{1}{2(\alpha+1)}}}\left[\left(\frac{B}{R}\right)^{3(\gamma+1)(\alpha+1)}-A\right]^{\frac{1}{2(\alpha+1)}}
\end{aligned}
$$

When the cosmological radius tends to the critical value of R, i.e. $R^{*}=\frac{B}{A^{\frac{1}{3(\gamma+1)(\alpha+1)}}}$, the energy density disappears and the pressure according to the equation of state grows indefinitely. Then cosmological time $t=t_{B}$. Again, when the cosmological radius grows indefinitely, i.e., $\mathrm{R} \rightarrow \infty$, then the energy density does not disappear and had some parametric value as $\dot{R} \rightarrow \infty$ (i.e., $\dot{R}_{I} \rightarrow \infty$, then $R_{I}=R_{I}^{*}=i R^{*}$ in other phase), then

$$
\rho(R)=\frac{1}{(\gamma+1)^{1 /(\alpha+1)}}[-A]^{\frac{1}{1+\alpha}}
$$

As well as the pressure is then

$$
\begin{aligned}
& p=\frac{\gamma}{(\gamma+1)^{1 /(\alpha+1)}}[-A]^{\frac{1}{1+\alpha}}+A \cdot \frac{1}{(\gamma+1)^{\frac{\alpha}{\alpha+1}}}[-A]^{-\frac{\alpha}{\alpha+1}} \\
& p(R)=\frac{A}{[-A]^{\alpha /(\alpha+1)} \cdot(\gamma+1)^{1 /(\alpha+1)}} \text { is negative for } \mathrm{A}\langle 0, \gamma>0, \alpha>0 .
\end{aligned}
$$

Hence, $\quad \rho+3 p=\frac{2 A}{(\gamma+1)^{1 /(\alpha+1)}[-A]^{\alpha /(\alpha+1)}}$

is not real for $\mathrm{A}>0, \alpha>0, \gamma>-1$ and $\rho+3 p \ngtr 0$ when $\mathrm{A} \ngtr 0$.

\section{Unified Field: SU (11)}

In the theory of Gauss transformation in physics, the special unitary group is used to represent bosonic symmetries. In the theories of symmetry breaking, it is important to find the subgroups of special unitary group. Important subgroups of SU(n) that are important in GUT physics (also in the present dissertation). Super unified theory are

For $\quad \mathrm{p}>1, \mathrm{n}-\mathrm{p}>1$ with $\mathrm{SU}(\mathrm{n}) \supset \mathrm{SU}(\mathrm{p}) \times \mathrm{SU}(\mathrm{n}-\mathrm{p}) \times \mathrm{U}(1)$.

For completeness there are also the orthogonal and sympletic subgroup: $\quad \mathrm{SU}(\mathrm{n}) \supset \mathrm{O}(\mathrm{n}) ; \quad \mathrm{SU}(2 \mathrm{n}) \supset \mathrm{US} p(2 \mathrm{n})$ 
Since the rank of $\mathrm{SU}(\mathrm{n})$ is $\mathrm{n}-1$ and $\mathrm{U}(1)$ is 1 , a useful check is that the sum of the ranks of the subgroups is less than or equal to the rank of original group $\mathrm{SU}(\mathrm{n})$ which is a subgroup of various other lie group : $\quad \mathrm{SO}(2 n) \supset \mathrm{SU}(\mathrm{n})$. From the symmetry breaking of $\mathrm{SU}(11)$, we find $\mathrm{SU}(6)$ and $\mathrm{SU}(5)$ as the subgroups of $\mathrm{SU}(11)$, where $\mathrm{p}(=5)>1 ; \mathrm{n}-\mathrm{p}(=11-5=6)>1$, so that $\mathrm{SU}(\mathrm{n}) \supset \mathrm{SU}(\mathrm{p}) \times \mathrm{SU}(\mathrm{n}-\mathrm{p}) \times \mathrm{U}(1)$. i.e. $\mathrm{SU}(11) \supset$ $\mathrm{SU}(5) \times \mathrm{SU}(6) \times \mathrm{U}(1)$. For completeness there are also the orthogonal and sympletic subgroups:

$\mathrm{SU}(11) \supset \mathrm{O}(11) ; \mathrm{SU}(22) \supset \mathrm{US} \mathrm{p}(22)$.

Since the rank of $S U(11)$ is 10 and $U(1)$ is 1 , a useful check is that the sum of the ranks of the subgroup $\mathrm{SU}(5)$ and $\mathrm{SU}(6)$ is less than or equal to the rank of the original group. Thus, we have from SU(11), the Hermitian matrix $\mathrm{H}$ has 120 arbitrary constants. Which correspond to 120 bosons that now mediate between the different basic entities. Of these we already have 24 from SU(5) and 35 from SU(6) and 1 from U(1).

Thus, $120-(24+35+1)=60$ more bosons are needed to make up the list of 120 . For want of any specific designation, they are referred to simply as the $\mathrm{J}$ bosons. The $\mathrm{J}$ bosons are expected to link the participants of $\mathrm{SU}(6)$ with $\mathrm{SU}(5)$ i.e. with $\mathrm{SU}(2), \mathrm{SU}(3)$ and $\mathrm{U}(1)$. There are emitted and absorbed $\overline{\mathrm{J}}$ particles (anti-J particles). Therefore, in the theory of SU(11), it is possible to change any of 30(thirty) latent energy bosons of SU(6) into any of the 30(thirty) matter energy bosons of SU(5) or vice-versa by the exchange of the Jbosons of SU(11). So at this stage, by the symmetry breaking of SU(11) created an amount of matter energy $\mathrm{SU}(5)$ by the latent energy group SU(6), and an angular momentum ' $\mathrm{J}$ ' \& Schwarzschild mass ' $\mathrm{M}$ ' of the blackhole by the energy group $\mathrm{U}(1)$. After then $\mathrm{SU}(5)$ breaks into $\mathrm{SU}(3) \times \mathrm{SU}(2)_{\mathrm{L}} \times \mathrm{U}(1)$.

\section{Intelligence: SU (6)}

In the transformations under the energy group SU(6), the basic fields here are the latent energy field and we have

$$
\mathrm{U}=\exp (-\mathrm{iH})
$$

Where ' $\mathrm{H}$ ' is a $6 \times 6$ Hermitian matrix of zero-trace. The matrix $\mathrm{H}$ now has 35 independent components. In the weak interaction $\mathrm{SU}(2)$, we have, $\mathrm{H}$ has $2 \times 2$ Hermitian matrix of zero-trace and the most general form of such matrix is

$$
H=\left(\begin{array}{cc}
a & b+i c \\
b-i c & -a
\end{array}\right)=a\left(\begin{array}{cc}
1 & 0 \\
0 & -1
\end{array}\right)+b\left(\begin{array}{ll}
0 & 1 \\
1 & 0
\end{array}\right)+c\left(\begin{array}{cc}
0 & i \\
-i & 0
\end{array}\right)
$$

Thus, like above, we have 35 matrix charges $\mathrm{I}_{1}, \mathrm{I}_{2}, \mathrm{I}_{3}, \ldots \ldots \ldots . . \mathrm{I}_{35}$ out of which five matrices are diagonal. Corresponding to this, we have 35 bosons. For want of any specific designation, they are referred to simply as $\mathrm{J}_{\mathrm{k}}$. There were no change takes place for exchanging the bosons namely $\mathrm{J}_{\mathrm{k} 3}, \mathrm{~J}_{\mathrm{k} 8}, \mathrm{~J}_{\mathrm{k} 15}, \mathrm{~J}_{\mathrm{k} 24}, \mathrm{~J}_{\mathrm{k} 35}$, corresponding to the said five diagonal matrices. We expect the participating interactions of the bosons $\mathbf{J}_{\mathrm{k}}$ to have comparable strength. The $\mathrm{J}_{\mathrm{k}}$ bosons are expected to generate a latent force. This force is believed to be potentially so large that the exotic matter fluid are expected to transfer into the ordinary matter field constituting a black-hole at the centre region.

\section{Super Unified Field: SU (23)}

In the theory of Gauss transformation in physics, the special unitary group is used to represent bosonic symmetries. In the theories of symmetry breaking, it is important to find the subgroups of special unitary group. Important subgroups of SU(n) that are important in GUT physics(also in the present dissertation). Super unified theory are

$$
\text { For } \quad \mathrm{p}>1, \mathrm{n}-\mathrm{p}>1 \quad \text { with } \quad \mathrm{SU}(\mathrm{n}) \supset \mathrm{SU}(\mathrm{p}) \times \mathrm{SU}(\mathrm{n}-\mathrm{p}) \times \mathrm{U}(1) \text {. }
$$

For completeness there are also the orthogonal and sympletic subgroup: $\quad S U(n) \supset O(n) ; S U(2 n) \supset$ US p (2n)

Since the rank of $\mathrm{SU}(\mathrm{n})$ is $n-1$ and $\mathrm{U}(1)$ is 1 , a useful check is that the sum of the ranks of the subgroups is less than or equal to the rank of original group $\mathrm{SU}(\mathrm{n})$ which is a subgroup of various other lie group : $\mathrm{SO}(2 \mathrm{n}) \supset \mathrm{SU}(\mathrm{n})$.

From the symmetry breaking of $\mathrm{SU}(23)$, we find $\mathrm{SU}(12)$ and $\mathrm{SU}(11)$ as the subgroups of $\mathrm{SU}(23)$, where $\mathrm{p}(=$ 11) >1; $\mathrm{n}-\mathrm{p}(=23-11=12)>1$, so that $\mathrm{SU}(\mathrm{n}) \supset \mathrm{SU}(\mathrm{p}) \times \mathrm{SU}(\mathrm{n}-\mathrm{p}) \times \mathrm{U}(1)$. i.e. $\mathrm{SU}(23) \supset \mathrm{SU}(12) \times \mathrm{SU}(11) \times$ $\mathrm{U}(1)$. For completeness there are also the orthogonal and sympletic subgroups:

$$
\mathrm{SU}(23) \supset \mathrm{O}(23) ; \mathrm{SU}(46) \supset \mathrm{US} \mathrm{p}(46) \text {. }
$$

Since the rank of $\mathrm{SU}$ (23) is 22 and $\mathrm{U}(1)$ is 1 , a useful check is that the sum of the ranks of the subgroup $\mathrm{SU}(12)$ and $\mathrm{SU}(11)$ is less than or equal to the rank of the original group. Thus, we have from $\mathrm{SU}(23)$, the Hermitian matrix $\mathrm{H}$ has 528 arbitrary constants. Which correspond to 528 bosons that now mediate between the different basic entities. Of these we already have 143 from SU(12) and 120 from SU(11) and 1 from U(1). 
Thus, $528-(143+120+1)=264$ more bosons are needed to make up the list of 528. For want of any specific designation, they are referred to simply as the $\mathrm{J}_{\mathrm{N}}$ bosons. The $\mathrm{J}_{\mathrm{N}}$ bosons are expected to link the participants of SU(12) with SU(11) i.e. with SU(6), SU(5) and hence SU(2), SU(3) and U(1). There are emitted and absorbed $\overline{J_{N}}$ particles (anti $J_{N}$ particles). Therefore, in the theory of $S U$ (23), it is possible to change any of 132 (one hundred and thirty two) latent energy bosons of SU(12) into any of the 132 (one hundred and thirty two) bosons of SU(11) or vice-versa by the exchange of the $\mathrm{J}_{\mathrm{N}}$-bosons of $\mathrm{SU}(23)$. So at this stage, by the symmetry breaking of SU(23) an amount of energy of SU(11) created for our universe, by the latent energy group SU(12).

\section{Intelligence: SU(12)}

In the transformations under the energy group SU(12), the basic fields here are the latent energy field and we have

$$
\mathrm{U}=\exp (-\mathrm{iH})
$$

Where ' $\mathrm{H}$ ' is a $12 \times 12$ Hermitian matrix of zero-trace. The matrix $\mathrm{H}$ now has 143 independent components. In the weak interaction $\mathrm{SU}(2)$, we have, $\mathrm{H}$ has $2 \times 2$ Hermitian matrix of zero-trace and the most general form of such matrix is

$$
H=\left(\begin{array}{cc}
a & b+i c \\
b-i c & -a
\end{array}\right)=a\left(\begin{array}{cc}
1 & 0 \\
0 & -1
\end{array}\right)+b\left(\begin{array}{ll}
0 & 1 \\
1 & 0
\end{array}\right)+c\left(\begin{array}{cc}
0 & i \\
-i & 0
\end{array}\right)
$$

Thus, like above, we have 143 matrix charges $\mathrm{I}_{1}, \mathrm{I}_{2}, \mathrm{I}_{3}, \ldots \ldots \ldots . ., \mathrm{I}_{143}$. Corresponding to this, we have 143 bosons. For want of any specific designation, they are referred to simply as $\mathrm{J}_{\mathrm{kn}}$. We expect the participating interactions of the bosons $\mathrm{J}_{\mathrm{kn}}$ to have comparable strength. The $\mathrm{J}_{\mathrm{kn}}$ bosons are expected to generate a latent force. This force is believed to be potentially so large that the energy like as gas are expected to transfer into the energy like vapour for the construction of our universe.

In the same process, we can explain the intelligence /consciousness of the latent energy group SU(24) and super unified field SU(47) etc. and so on, up-to the Big-Rip singularity.

Hence, if we want to express all the sub-sets of the energy groups into a single frame-work like GUT physics, we get a unified group (absolute vacuum, i.e. so called nothing) $\mathrm{SU}(\infty)$, whose $\mathrm{J}_{\infty}$ bosons of $\mathrm{SU}(\infty)$ are expected to link the participants of $\mathrm{SU}(\infty) \times \ldots \ldots \ldots \ldots \ldots \ldots . \mathrm{SU}(24) \times \mathrm{SU}(12) \times \mathrm{SU}(6) \times \mathrm{SU}(3) \times \mathrm{SU}(2) \times \mathrm{U}(1)$.

Therefore, it is shown that according to the quantum theory, there were no singularities at all, only the process of condensation, causes the symmetry breaking of the energy group starting from infinite space-time i.e. Big-Rip singularity (see, THE COMPLEX QUANTUM AND CLASSICAL PSEUDO-TACHYONIC UNIVERSE), where the energy pressure and density exists, depending on some arbitrary values. Actually our material universe starts from a unified single frame-work of the energy group SU(11) by the symmetry breaking of the energy group SU(23) and similarly all other energy groups created by the symmetry breaking of the energy group from infinity, where the energy group other than SU(5), are responsible for the consciousness, intelligence and other activities of the living cells depending on the progress of several biological revolution which also depends on SU(5). Whereas SU(5) contributes to the material building of living cells as well as inanimate objects also of this Universe. Most of these energy groups are unfolded and activate in the humanlike living cells. This type of energies create some images (Physical and artificial-from the sub-conscious level of mind) with different frequencies, which never die at all and bounce it with the return to the same frequencies energy waves within the living cell. An analogy will illustrate the scenario. Suppose a message is created and will be sent from an observer's mobile to another observer or observer's mobile. If we consider the receiver's mobile was switched-off, then the message wave will fly till the receiver's mobile is switched on and as soon as the mobile is switched on, the observer will receive the message immediately without tempering it, if the other conditions remain unaltered. Otherwise the message will be hampered. The same will be compared with the consciousness or intelligence energy waves with different frequencies as message for living cells within the span of life like switched on mobile of the observer.

\section{Concluding Remarks: Consciousness in the Universe.}

Our criterion for proto-consciousness is OR. It would be unreasonable to refer to OR as the criterion for actual consciousness, because, according to the DP scheme, OR processes would be taking place all the time, and would be providing the effective randomness that is characteristic of quantum measurement. Quantum superposition will continually be reaching the DP threshold for OR in non-biological setting as well as in biological ones, and usually take place in the purely random environment of a quantum system under measurement. A possible remarkable change within the existing living cell can be made by the energy of consciousness and the environment namely the disease cancer etc. So cancer etc. can be cure by using this energy therapy. 
To find the quantum measurement of the universe, it is necessary to do beyond the concept of standard model of physics as well as the Einstein universe. We need a new concept with the Einstein's universe and the standard model of physics to proceed any further, and Einstein's general relativity with complex space $\left(R+i R_{I}\right)$ of $(4+$ D) - dimension, D is an extra dimension or a generalized Gaussian energy group is one of such theory. We introduce an infinite number of new energy sources which are actually responsible for the consciousness and intelligence of the universe and also the living element. It was shown previously, in my published paper [Bhadra NK; THE COMPLEX QUANTUM AND CLASSICAL PSEUDO-TACHYONIC UNIVERSE; (IOSR-JM) eISSN: 2278-5728,p-ISSN: 2319-765X, Volume 8, Issue 3 (Sep. - Oct. 2013), PP 15-32] that at the Big-Rip singularity, where the space-time will be infinite but the energy density and pressure must exist and depends on some arbitrary constant. According to quantum mechanics, it seems to us to the universe in four segments, one from infinite to 10-dimensional space-time, then from 10-dimensional to 7-dimensional flat universe, then Black-hole stage up-to 4-dimensional then our 4-dimensional Einstein's universe. An analogy will illustrate the scenarios with gas-vapor-liquid state, where below the critical temperature, gas becomes vapor, and then easily changes to liquid, similarly the constructing process of our universe has started from $\mathrm{SU}(23)$. Actually there exists only two singularities, one, staring from Big-Rip singularity to Big-Brake through Big-Bang singularity and from Big-Brake singularity to Big-Rip through Big-Crunch singularity under the contraction of the universe. Hence we conclude the action of new energy source created by the symmetry breaking of energy group from infinite must [i.e. actual vacuum space (nothing) is the source of all kinds of energies (i.e. it is not empty, fill-up with some-kind new energy sources)] activate within the universe and control the universe including the living elements, we can not escape from this universe separately without vanishing the universe as a whole.

\section{Acknowledgement:}

The author is thankful to Prof. Subenoy Chakraborty, Department of Mathematics, Jadavpur University, Kolkata, West Bengal, India, for his helpful discussions.

\section{References:}

[1]. Everett, H. (1957). Relative state formulation of quantum mechanics. In Quantum Theory and Measurement, Wheeler, J.A., and zurek,W.H., (eds) Princeton University Press, 1983; Originally in Reviews of Modern Physics, 29, 454.

[2]. Engel, G.S., Calhoun, T.R., Read, E.L., Ahn, T-K., Mancal, T., Cheng, Y-C., Blankenship, R.E., Fleming, G.R., (2007). Evidence for wave-like energy transfer through quantum coherence in photo synthetic systems. Nature 446: 782.

[3]. Bhadra N.K(2012):The complex Model of the Universe, IOSR-JM, ISSN: 2278-5728, vol.2, 4, pp-20; and The complex model of the quantum universe, ISSN: 2278-5728. IOSR Journal of Mathematics (IOSR-JM) vol.4, Iss. 1 (Nov. - Dec 2012), pp-20. 'The Complex Quantum-State of Black-Hole and Thermostatistics' (IOSR-JM: e-ISSN: 2278-3008, p-ISSN: 2319-7676. Volume 8, Issue 5 (Nov. - Dec. 2013), PP 01-19)].

[4]. Bhadra NK; THE COMPLEX QUANTUM AND CLASSICAL PSEUDO-TACHYONIC UNIVERSE; (IOSR-JM) e-ISSN: 22785728,p-ISSN: 2319-765X, Volume 8, Issue 3 (Sep. - Oct. 2013), PP 15

[5]. Einstein, A. de-Sitter, W. (1932): On the relation between the expansion and mean density of the universe. Proc. Natl. Acad. Sci.,(USA), 18,213. Hawking S.W(1984): The quantum state of the universe, Nucl. Phys. B239.257.

[6]. Davies, P.C.W. (1974): The Physics of Time Asymmetry(Surrey University Press/ University of California Press) - 1976a Proc. R. Soc. A. 351 139; -1976b Nature 263 377; -1977a Proc. R. Soc. A. 353 499; -1977b Space and Time in the Modern University (Cambridge : Cambridge University Press).

[7]. Bandyopadhyaya, A (2011) Direct experimental evidence for quantum states in microtubules and topological invariance. Towards a science of consciousness 2011, Sockhalm, Sweden, HYPERLINK 'http://www.consciousness.arizona.edu.'

[8]. Ghirardi, G.C., Rimini, A., and Weber, T. (1986). Unified dynamics for microscopic and microscopic systems. Physical Review D, 34, 470. Ghirardi, G.C., Rimini, A., and Grassi, R. (1990). Continuous spontaneous reduction model involving gravity. Physical Review A, 42, 1057.

[9]. Candelas P., and Seiama, D.W. 1977 Phys. Rev. Lett. 38 1372; Carr, B. 1977 Mon, Not. R. Astrom. Soc. 181 293; Carter B 1973 Black-Holes ed DeWitt and DeWitt (London: Gordon and Breach) Casimir HBG 1948 Proc. Kon. Ned. Akad. Wetens chap. 51793.

[10]. Christensen, S.M. and Fulling, S.A. 1977 Phys. Rev. D15 2088; Einstein Albert (1987): Ideas and Opinions, Crown Publishers, New York, pp-348.

[11]. Halliwel, J.J. Hawking, S.W., (1985): The Origin of Structure in the Universe, Phys. Rev. D 31 1777. Hawking, S.W.(1985): The Arrow of Time in Cosmology Phys. Rev., D 32 2489. 1.

[12]. Bennett, C.H., and Wiesner, S.J.(1992). Communication via-1and 2-particle operators on Einstein-Podolsky-Rosen states. Physical Review Letters, 69, 2881-84.

[13]. Bensimon. G.,Chemat, R. (1991). Microtubule disruption and cognitive defects: effects of colchicines on teaming behavior in rats. Pharmacol. Biochem. Behavior 38: 141-145. Atema, J. (1973). Microtubule theory of sensory transduction. Journal of theoretical Biology, 38, 181.

[14]. Guth, A. and Steinhardt. Paul(1992): The inflationary Universe and article in The New Physics edited by Paul Davies (Cambridge university Press). Pp-55. Smarr L 1973 Phys. Rev. Lett. 3071.

[15]. Appelquist, T., Chodos, A. and Freund, P.G.O (1986): Modern Kaluza-Klein Theories, Frontiers in Physics in series Vol. 65, (Ed. Adison-Wesley). A.G. Riess et al, Astrophys ., 560,49(2001). DeWitt, B.S., Phys Rev 160, 1113 (1967).

[16]. Abdul Rahaman, A.M.M.(1992): Phys.Rev. D 45, 3497; Abbott, L.F. (1985): Phys. Lett. 150B, 427. Giddings, S.B. and Strominer, A. (1988-89): Nucl. Phys., B 307, 854 B 321, 481(1989).

[17]. Emmy Noether, Gesammette Abhandlungen, (Collected Papers), Springer-Verlag, Berlin-New-York, 1983. Wigner, E. 'Symmetry and Conservation Laws', Physics Today, March 1964 P.34. Anderson, P.W., pp 175-182 in concepts in solids (W.A. Benjamin Inc., New-York, 1964). 
[18]. Parratt, L.G., Probability and Experimental Errors in Science, Wiley, New-York, 1961 and Parzen, E., Modern Probability Theory and Its Application, Wiley, New-York, 1960. C. Huang, Statistical Mechanics, Wiley, 1963. Classic graduate text. ; R.J.Finkelstein, Thermodynamics and Statistical Physics-A Short Introduction, W. H. Freeman and Co., 1969.

[19]. M. Tribus, Thermostatics and Thermodynamics, Van Nostrand, 1961. ; P. T. Landsburg, Thermodynamics and Statistical Mechanics, Oxford University Press, 1978. ; P.W. Anderson, Basic Notions of Condensed Matter Physics, Benjamin/Cummings, 1984. ; J. W. Gibbs, The Scientific Papers of J. Willard Gibbs, Volume I, Thermodynamics, Dover, 1961.

[20]. Bohm, D. (1951). Quantum Theory (Prentice-Hall, Englewood-Cliffs.) ch.22, Sect.15-19. Reprinted as: the paradox of Einstein, Rosen and Podolsky, in quantum Theory and Measurement, eds., J.A. Wheeler and Zurek, W.H. (Princeton University Press, Princeton, 1983).

[21]. Bernroider, G. and Roy, R. (2005) Quantum entanglement of k-ions, multiple channel states and the role of noise in the brain. SPIE 5841-29: 205. Bouwmester, D., Pan, J.W., Mattle, K., Eibl, M. , Weinfurter, H. and Zeilinger, A. (1997). Experimental quantum teleportation. Nature, 390(6660):575.

[22]. Chalmars, D.J. (1996). The conscious mind- In search of a fundamental theory. Oxford University Press, New York. Crick, F. , and Koch, C,(1990). Towards a neurobiological theory of consciousness. Seminars in the Neuroscience,2, 263. Dennett, D.C.(1991)consciousness explained. Little Brown, Boston, M.A (1995)- Darwin's dangerous idea : Evolution and the Meaning of Life, Simpon and Schuster.

[23]. Dcutsch, D. (1985) Quantum theory, the Chureh-Turing principle and the universal quantum computer, Proceedings of the Royal Society (London) A 400, 97. Benioff, P.(1982). Quantum mechanical Hamiltonian models of Turing mechanics. Journal of statistical physics, 29.

[24]. Dio' si, L. (1987). A universal master equation for the gravitational violation of quantum mechanics, Physics Letters A 120(8): 377 and Dio' si, L. (1989) Models for universal reduction of microscopic quantum fluctuation. Physical Review A, 40, 1165.

[25]. Draguhn, A., Traub, R.D., Schmitz, D., Jeffery, S.(1998). Electrical coupling underlies high frequency oscillations in the hippocampus in vitro. Nature, 394(6689), 189. Eccles, J.C.(1992). Evolution of consciousness. Proceedings of the National Academy of Sciences, 89, 7320 .

[26]. Feynman, R.P.(1986). Quantum mechanical computers. Foundations of physics, 16(6), 507. Grush, R., Churchland, D.S., (1995). 'Gaps in Penrose's toilings', J. Consciousness studies, 2(1): 10. Koch, C.., Hepp, K.(2006). Quantum mechanics in the brain. Nature 440(7084): 611. Hameroff, S.R., and Penrose, R(1996b). conscious events as orchestrated space-time selections. J. Consciousness studies, 3(1): 36. Koch, C.(2004). The Quest for consciousness: A Neurobiological Approach, Englewood, co., Roberts and co., Koch, C., Hepp, K. (2006). Quantum mechanics in the brain. Nature 440(7084): 611.

[27]. Hagan, S., Hameroff, S., and Tuszynski, J. (2001). Quantum computation in Brain Microtubules? Decoherence and Biologica Feasibility, Physical Review E, 65, 061910. Hameroff, S.R., and Penrose, R., (1996a). Orchestrated reduction of quantum coher ence in brain microtubules: A model for consciousness. In: Towards a science of consciousness: The first Tucson Discussions and Debates. Hameroff, S.R., Kaszniak, and Scott, A.C.eds., MIT Press, Cambridge M A., 507. Also published in Mathematics and Computers in simulation (1996). 40: 453.

[28]. Hameroff, S., (1998a). Quantum computation in brain microtubules? The Penrose-Hameroff 'Orch OR' model of consciousness. Philosophical Transcations of the Royal Society (London) Series A. 356,1869. Hameroff, S., (1998b). 'Fundamentality': is the conscious mind subtly linked to a basic level of the universe? Trends in Cognitive Science, 2, 119.

[29]. Marshall, W., Simon, C., Penrose, R., and Bouwmeester, D.(2003). Towards quantum superposition of a mirror. Physical Review Letters 91, 13.; 130401. Mckemmish, L.K., Reimers, J.R., Mckenzie, R.H., Mark, A.E., Hush, N.S.(2009). Penrose- Hameroff orchestrated objective-reduction proposal for human consciousness is not biologically feasible. Physical Review E. 80(2 Pt 1): 021912 .

[30]. Moroz, I.M., Penrose, R., and Tod, K.P.(1998). Spherically-symmetric solutions of the Shrödinger - Newton equation : Classical and Quantum Gravity, 16, 2733, Pearle, P. and Squires, E.J.(1994). Bound-state excitation, nucleon decay experiments and models of wave-function collapse. Physical Riview Letters, 73(1), 1-5.

[31]. Penrose, R.(1989). The Emperor's New Mind: Concerning Computers, Minds, and the Laws of physics, Oxford University Press, Oxford. Penrose, R(1994). Shadows of the Mind; An Approach to the Missing Science of consciousness. Oxford University Press, Oxford. Penrose, R(1996). On gravity's role in quantum state reduction. General Relativity and Gravitation, 28, 581.

[32]. Polkorny, J.(2004). Excitation of vibration in microtubules in living cells. Bioelectrochem. 63: 321. Samsonovich, A., Schott, A., Hameroff, S.(1992). Acousto-Conformational transition in cytoskeletal microtubules : Implications for intracellular information processing. Nanobiology 1: 457. Consciousness in the universe; Penrose, R and Hmeroff, S. Journal of Cosmology, 2011, vol.14.

[33]. B.S. DeWitt, Phys Rev 160, 1113 (1967); C. Kiefer, Quantum Gravity (Oxford University Press, Oxford, 2007), $2^{\text {nd }}$ ed; A. Riess et al., Astron J116, 1009 (1998). 